\title{
PoWR grids of non-LTE model atmospheres for OB-type stars of various metallicities
}

\author{
R. Hainich ${ }^{1}$, V. Ramachandran ${ }^{1}$, T. Shenar ${ }^{1,2}$, A. A. C. Sander ${ }^{1,3}$, H. Todt $^{1}$, D. Gruner ${ }^{1}$, \\ L. M. Oskinova ${ }^{1}$, and W.-R. Hamann ${ }^{1}$
}

\author{
1 Institut für Physik und Astronomie, Universität Potsdam, Karl-Liebknecht-Str. 24/25, 14476 Potsdam, Germany \\ e-mail: rhainich@astro.physik.uni-potsdam.de \\ 2 Institute of astrophysics, KU Leuven, Celestijnlaan 200D, 3001 Leuven, Belgium \\ 3 Armagh Observatory and Planetarium, College Hill, Armagh BT61 9DG, UK
}

Received 6 July 2018 / Accepted 5 November 2018

\begin{abstract}
The study of massive stars in different metallicity environments is a central topic of current stellar research. The spectral analysis of massive stars requires adequate model atmospheres. The computation of such models is difficult and time-consuming. Therefore, spectral analyses are greatly facilitated if they can refer to existing grids of models. Here we provide grids of model atmospheres for OB-type stars at metallicities corresponding to the Small and Large Magellanic Clouds, as well as to solar metallicity. In total, the grids comprise 785 individual models. The models were calculated using the state-of-the-art Potsdam Wolf-Rayet (PoWR) model atmosphere code. The parameter domain of the grids was set up using stellar evolution tracks. For all these models, we provide normalized and flux-calibrated spectra, spectral energy distributions, feedback parameters such as ionizing photons, Zanstra temperatures, and photometric magnitudes. The atmospheric structures (the density and temperature stratification) are available as well. All these data are publicly accessible through the PoWR website.
\end{abstract}

Key words. stars: massive - stars: early-type - stars: atmospheres - stars: winds, outflows - stars: mass-loss - radiative transfer

\section{Introduction}

Through their powerful stellar winds, ionizing fluxes, and supernova (SN) explosions, massive stars $\left(M_{\mathrm{i}} \gtrsim 8 M_{\odot}\right)$ dominate the energy budget of their host galaxies. They are the progenitors of core-collapse $\mathrm{SNe}$, leaving behind a neutron star (NS) or a black hole $(\mathrm{BH})$, which makes them central players in modern gravitational-wave (GW) astrophysics (e.g., Marchant et al. 2016; de Mink \& Mandel 2016; Hainich et al. 2018). Spectroscopically, they are predominantly identified with $\mathrm{O}$ and early B spectral types. When surrounded by thick stellar winds, they are classified as Wolf-Rayet (WR) stars (Smith 1968; Smith et al. 1996), as transition-type stars, such as Of/WN stars (e.g., Crowther \& Walborn 2011), or as luminous blue variables (LBVs; e.g., Humphreys \& Davidson 1994; van Genderen 2001)

In recent years, the topic of massive stars at low metallicity $(Z)$ has been gaining tremendous momentum. The first stars that formed in our universe must have been born in extremely $Z$-poor environments (Bromm \& Larson 2004). Massive binaries at low $Z$ are the leading candidates for massive GW merger systems (e.g., Eldridge \& Stanway 2016). Generally, massive stars as a function of $Z$ are intensively studied; for example, the $Z$-dependence of multiplicity parameters (Sana et al. 2013; Almeida et al. 2017), initial masses (Schneider et al. 2018), binary interaction physics (Foellmi et al. 2003; Shenar et al. 2016, 2017), stellar feedback (Ramachandran et al. 2018a,b), stellar rotation (Meynet \& Maeder 2005), and stellar winds (Mokiem et al. 2007; Hainich et al. 2015). The Small and Large Magellanic Clouds (SMC, LMC), with their well-constrained distances, low interstellar extinctions, and subsolar metallicity of $\sim 1 / 7$ and $1 / 2$ solar, respectively (Dufour et al. 1982;
Larsen et al. 2000; Trundle et al. 2007), offer ideal laboratories for studying $Z$-dependent effects.

The physical parameters of massive stars, such as their temperatures, luminosities, and masses, can be derived by comparing observed to synthetic spectra. To model massive star atmospheres, it is essential to allow for non-local thermodynamic equilibrium (non-LTE), and to account for the millions of iron-line transitions in the ultraviolet (UV) that give rise to the so-called line-blanketing (e.g., Hubeny \& Lanz 1995; Hillier \& Miller 1998). For most O-type stars, as well as for evolved B-type stars, a calculation of the wind is also required (Hamann 1981; Kudritzki et al. 1992; Puls et al. 2008). There are only a few codes worldwide that fulfill these requirements (see overviews in, e.g., Puls et al. 2008; Sander et al. 2015).

The Potsdam Wolf-Rayet (PoWR) model atmosphere program is one of these codes. Originally developed for WR stars, it is now applicable to any hot star that does not show significant deviations from spherical symmetry, including OB-type stars (Gräfener et al. 2002; Hamann \& Gräfener 2003; Sander et al. 2015). Using PoWR, fundamental parameters have been derived for many WR stars and binaries in the Galaxy (Hamann et al. 1995; Sander et al. 2012) and the Magellanic Clouds (Hainich et al. 2014; Shenar et al. 2016), as well as for OB-type stars and binaries (Shenar et al. 2015; Ramachandran et al. 2018a). PoWR model grids for WR stars of various types and at various metallicites have been published online ${ }^{1}$ (Sander et al. 2012; Hamann \& Gräfener 2004; Todt et al. 2015). With the current paper, we announce the publication of extensive model grids of OB-type stars at SMC,

\footnotetext{
1 wWW.astro.physik. uni-potsdam.de/PoWR
} 


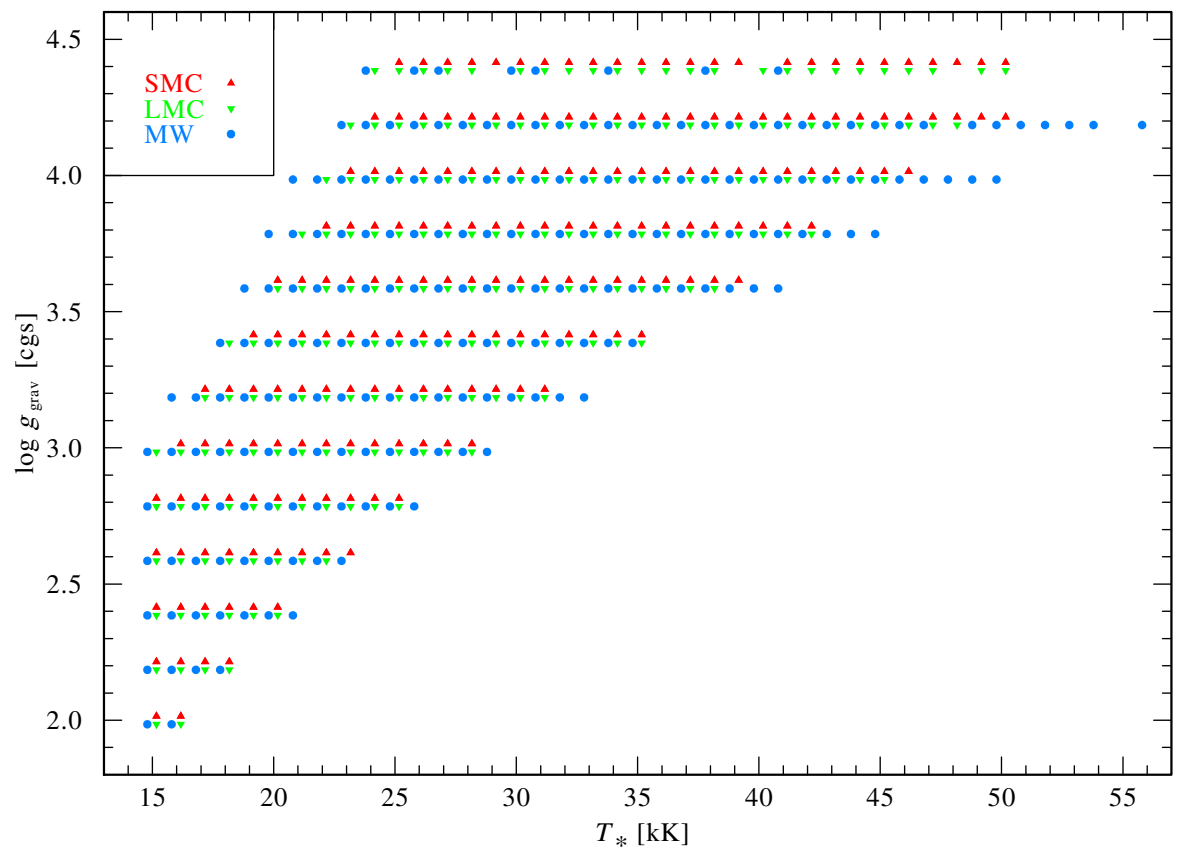

Fig. 1. Overview of OB-type model grids in the $T_{*}-\log g_{\text {grav }}$ plane. Each symbol represents an available PoWR model. The different colors and symbols indicate the different grids described in Sect. 3. The extension of the two SMC grids is identical.
LMC, and solar metallicities calculated with the PoWR code. The applicability of these model grids ranges from spectral analyses of OB-type stars to theoretical applications that need model spectra as an input such as population synthesis (e.g., Leitherer et al. 2014; Eldridge et al. 2017), or applications that require atomic level population numbers as input such as threedimensional (3D) Monte-Carlo calculations of stellar winds (e.g., Šurlan et al. 2012a,b).

The paper is structured as follows. In Sect. 2 we describe the basics of the PoWR atmosphere models. The OB-type grids, the data products, and the web interface are introduced in Sect. 3. In Sect. 4 we discuss some findings based on our model calculations. Finally, we give a short overview of potential applications in Sect. 5.

\section{The models}

The synthetic spectra presented in this work are calculated with the Potsdam Wolf-Rayet (PoWR) code, which is a state-ofthe-art code for expanding stellar atmospheres. PoWR assumes spherical symmetry and a stationary outflow. It accounts for nonLTE effects, a consistent stratification in the hydrostatic (lower) part of the atmosphere, iron line blanketing, and wind inhomogeneities. The code solves the rate equations for the statistical equilibrium simultaneously with the radiative transfer, which is calculated in the comoving frame. At the same time, the code ensures energy conservation. For details on the code, we refer to Gräfener et al. (2002), Hamann \& Gräfener (2003), Todt et al. (2015), and Sander et al. (2015).

The main parameters of OB-type models are the stellar temperature $T_{*}$, the luminosity $L$, the surface gravity $\log g_{\text {grav }}$, the mass-loss rate $\dot{M}$, and the terminal wind velocity $v_{\infty}$. The stellar temperature and the luminosity specify the stellar radius $R_{*}$ via the Stefan-Boltzmann law

$L=4 \pi \sigma_{\mathrm{SB}} R_{*}^{2} T_{*}^{4}$.

The stellar radius is by definition the inner boundary of the model atmosphere, which we locate at a Rosseland continuum optical depth of $\tau_{\text {Ross }}=20$. The stellar temperature $T_{*}$ is then the effective temperature that corresponds to $R_{*}$. The outer boundary is set to $R_{\max }=100 R_{*}$.

In the subsonic part of the stellar atmosphere, the velocity field $v(r)$ is calculated self-consistently such that a quasihydrostatic density stratification is obtained. A classical $\beta$-law (Castor \& Lamers 1979; Pauldrach et al. 1986)

$v(r)=v_{\infty}\left(1-\frac{R_{0}}{r}\right)^{\beta}$,

with $R_{0} \approx R_{*}$ is assumed in the wind, which corresponds to the supersonic part of the atmosphere. For the exponent, the value $\beta=0.8$ is assumed for all models (Kudritzki et al. 1989; Puls et al. 1996).

In the comoving-frame calculations, turbulent motion is accounted for by using Gaussian line profiles with a Doppler width of $30 \mathrm{~km} \mathrm{~s}^{-1}$. This choice is motivated by the requirement to limit the computation time; tests revealed that narrower line profiles during the comoving-frame calculations have very limited impact on the resulting stratification. In the hydrostatic equation, the turbulent pressure is taken into account by means of a microturbulent velocity $\xi$ (see Sander et al. 2015).

After an atmosphere model is converged, the synthetic spectrum, also denoted as emergent spectrum, is calculated by integrating the source function in the observers frame along emerging rays parallel to the line-of-sight. In this formal integral the Doppler velocity is decomposed into a depth-dependent thermal component and the microturbulent velocity, which is set to $\xi\left(R_{*}\right)=14 \mathrm{~km} \mathrm{~s}^{-1}$ at the base of the wind and grows proportional to the wind velocity up to a value of $\xi\left(R_{\max }\right)=0.1 v_{\infty}$.

Wind inhomogeneities are accounted for by assuming optically thin clumping. The clumping factor $D$ (which is the inverse of the volume filling factor, $f_{\mathrm{V}}=D^{-1}$ ) describes the over-density in the clumps compared to a homogeneous model with the same mass-loss rate (Hillier 1991; Hamann \& Koesterke 1998), while the interclump medium is considered to be void. We assume that clumping starts at the sonic point and reaches its maximum value $D=10$ at a stellar radius of $10 R_{*}$ (cf. Runacres \& Owocki 2002).

Detailed model atoms of $\mathrm{H}, \mathrm{He}, \mathrm{C}, \mathrm{N}, \mathrm{O}, \mathrm{Mg}, \mathrm{Si}, \mathrm{P}$, and $\mathrm{S}$ were included in the non-LTE calculations (see Table A.1). The 


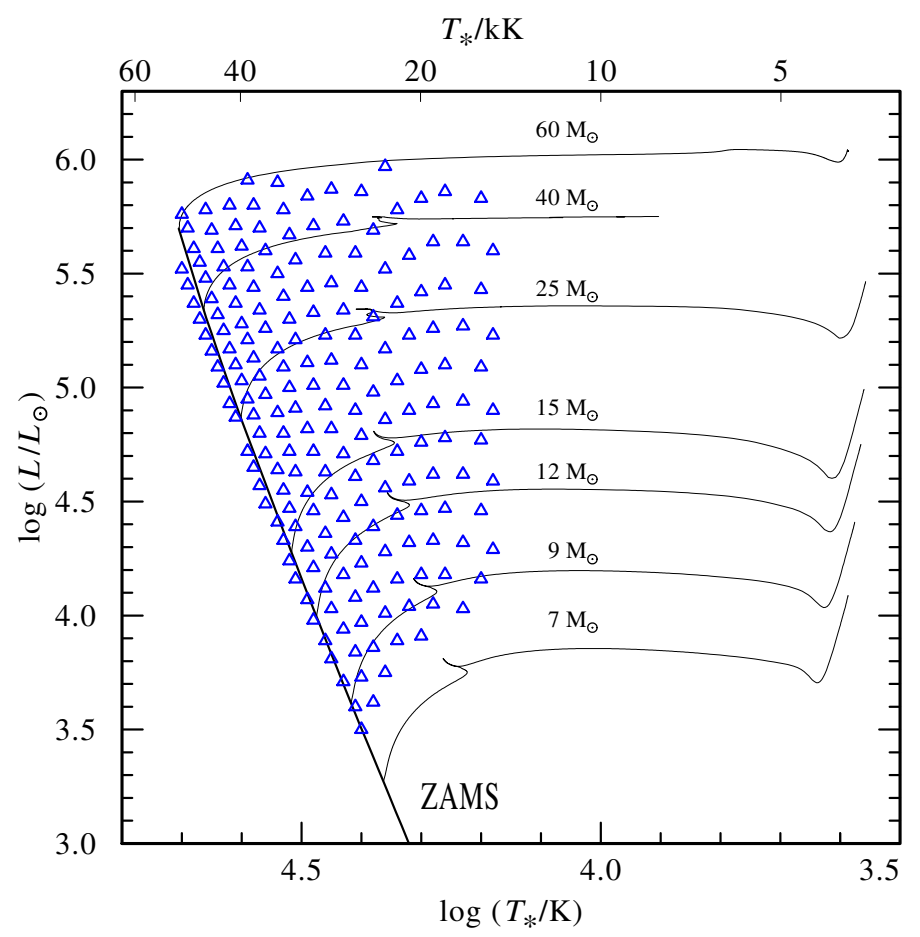

Fig. 2. Hertzsprung-Russell diagram illustrating the coverage of the $\log T_{*}-\log L$ domain by our SMC model-grid. Each blue triangle refers to one grid model. The depicted stellar evolution tracks were calculated by Brott et al. (2011).

iron group elements ( $\mathrm{Sc}, \mathrm{Ti}, \mathrm{V}, \mathrm{Cr}, \mathrm{Mn}, \mathrm{Fe}, \mathrm{Co}$, and Ni) with their multitude of levels and line transitions were treated in a superlevel approach (see Gräfener et al. 2002), combining levels and transitions into superlevels with pre-calculated transition cross-sections and with the assumption of solar abundance ratios relative to iron.

\section{The OB-type atmosphere grids}

The PoWR code is employed to construct grids of model atmospheres for early B-type and O-type stars. Altogether four grids have been calculated so far, ranging from solar metallicities down to $\mathrm{LMC}\left(Z_{\mathrm{LMC}} \approx 1 / 2 Z_{\odot}\right)$ and SMC metallicities $\left(Z_{\mathrm{SMC}} \approx 1 / 7 Z_{\odot}\right.$, Dufour et al. 1982; Larsen et al. 2000; Trundle et al. 2007). Two grids have been established for the SMC, which correspond to two different mass-loss rates, while only one grid has been calculated for the LMC and one for the Galaxy. The parameterization of the grids is illustrated in Fig. 1. In total 785 models have been calculated. Further grids that will improve the coverage of the mass-loss domain are currently in preparation and will be made available in the near future.

The independent parameters of the grid models are the stellar temperature $T_{*}$ and the surface gravity. The grid spacing is $1 \mathrm{kK}$ for $T_{*}$ and 0.2 dex for $\log g_{\text {grav }}$. The gravitational acceleration is given by

$g_{\text {grav }}=\frac{G M}{R_{*}^{2}}$

where $M$ is the stellar mass and $G$ the gravitational constant. For the SMC and LMC grids, models have been calculated for stellar temperatures of $15 \mathrm{kK}-50 \mathrm{kK}$, while the temperature range is $15-56 \mathrm{kK}$ for the Galactic grid. Besides $T_{*}$ and $\log g_{\text {grav }}$, the luminosity is a further model parameter. The value of $L$ has been set by using stellar evolution tracks and by interpolating among them. Because of that, the extension of the grids in the $\log g_{\text {grav }}$ domain is limited by the coverage of the stellar evolution tracks. This is illustrated in Fig. 2 that depicts a Hertzsprung-Russell diagram (HRD) with the SMC model grid and the stellar evolution models used to construct this grid. The corresponding plots for the other grids can be found in Appendix B. For the SMC and LMC grids, the stellar evolution models calculated by Brott et al. (2011) were employed, while the models by Ekström et al. (2012) were used for the Galactic grid, since those evolution models have a superior coverage of the initial mass domain. These different sets of evolution models are the reason why the extension of the grids is not the same for the MW, LMC, and the two SMC grids as visible in Fig. 1.

Based on $T_{*}, L$, and $\log g_{\text {grav }}$, the escape velocity for each model is calculated, which in turn is used to estimate the terminal wind velocity by applying the scaling relations established by Lamers et al. (1995). Accounting for the hot bi-stability jump, a factor of 1.3 is used for stars with $T_{*}<21 \mathrm{kK}$, while 2.6 is applied above $21 \mathrm{kK}$ (see also Lamers \& Cassinelli 1999). In addition, the terminal wind velocities for the SMC and LMC models are scaled with $\left(Z / Z_{\odot}\right)^{0.13}$, following Leitherer et al. (1992).

A further model parameter is the mass-loss rate $\dot{M}$ or, equivalently, the wind strength parameter $\log Q$, which is used instead of $\dot{M}$ in the two SMC grids to prescribe the wind mass-loss. In the PoWR code, the following definition of the $\log Q$ parameter is adopted

$Q=\frac{\dot{M} /\left(M_{\odot} \mathrm{yr}^{-1}\right) \cdot D^{1 / 2}}{\left(v_{\infty} /\left(\mathrm{km} \mathrm{s}^{-1}\right) \cdot R_{*} / R_{\odot}\right)^{3 / 2}}$,

(see e.g., Puls et al. 1996, 2008; Sander et al. 2017). The two SMC grids are calculated with $\log Q=-13.0$ and $\log Q=$ -12.0 , respectively. The use of a fixed $\log Q$ in those grids implies that the mass-loss rate is not constant throughout the grids, since $v_{\infty}$ and $R_{*}$ vary from model to model. In the MW and LMC grid, we instead used a fixed mass-loss rate of $\dot{M}=$ $10^{-7} M_{\odot} \mathrm{yr}^{-1}$ for all models. This value of $\dot{M}$ is chosen because our grids are meant as an extension of the parameter space of earlier grids, such as those published by Lanz \& Hubeny (2003, 2007), to significant mass-loss rates. Hence, a certain amount of wind is always present in our models. The models calculated by Lanz \& Hubeny $(2003,2007)$ with their TLUSTY code adopt the approximation of a plane-parallel and static atmosphere. Sander et al. (2015) showed that in the limit of vanishing $\dot{M}$ and infinite curvature radius, the emergent spectra of PoWR model atmospheres agree very well with the TLUSTY results.

The mass-loss rate is an import parameter that significantly determines the density in the wind and, consequently, also the emergent spectrum. The spectral range that is influenced the most by the choice of $\dot{M}$ is the UV with its key diagnostic wind lines such as $\mathrm{N}_{\mathrm{v}} \lambda \lambda 1239,1243 \AA$, Si Iv $\lambda \lambda 1393.8,1402.8 \AA$, $\mathrm{C}_{\text {IV }} \lambda \lambda 1548,1550.8 \AA$, He II $\lambda 1640 \AA$ and $\mathrm{N}_{\text {IV }} \lambda 1718 \AA$. Due to the choice of modest mass-loss rates for the presented grids, the emergent spectra of all models show at least some of those lines in the form of P Cygni profiles, depending on the specific ionization structure. In comparison to the UV, the optical wavelength range is significantly less influenced by mass loss. In this range, the main wind-contaminated lines are He II $\lambda 4686 \AA$ and $\mathrm{H} \alpha$. While $\mathrm{H} \alpha$ might show a certain amount of wind emission in its profile for the cool models with low surface gravities, the adopted mass-loss rates are usually too low to push He II $\lambda 4686 \AA$ into emission. Besides these two prominent lines, weaker nitrogen and carbon lines might appear in emission, as illustrated in 

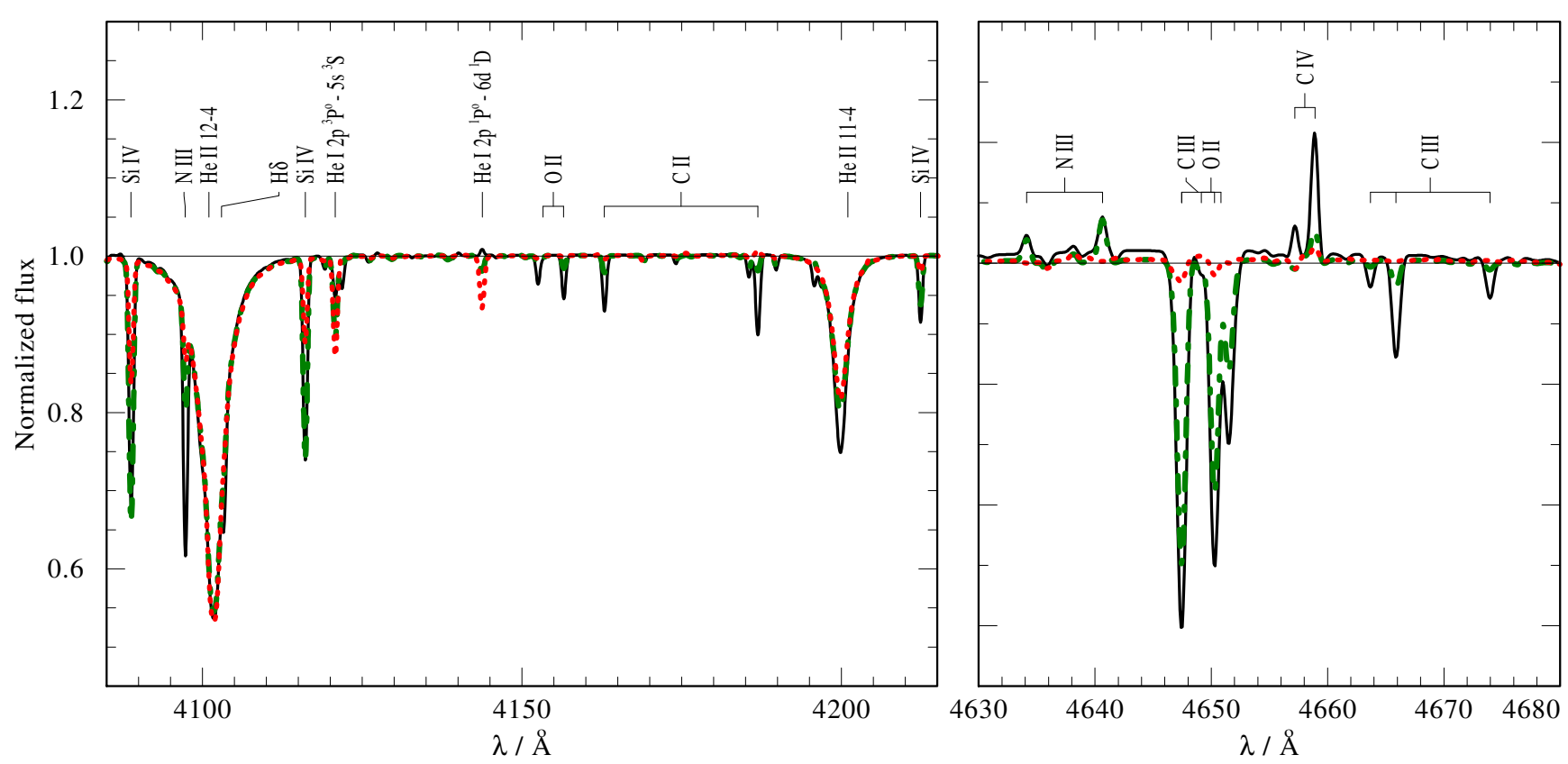

Fig. 3. Normalized line spectra of the models with $T_{*}=36 \mathrm{kK}$ and $\log g_{\text {grav }}=3.8$ [cgs] from the grid with SMC (red dotted line), LMC (green dashed line), and solar (black continuous line) metallicity. The mass-loss rate of all models is $\dot{M}=10^{-7} M_{\odot} \mathrm{yr}^{-1}$. Two exemplary wavelength ranges with prominent metal lines are depicted.

the right panel of Fig. 3. In the infrared (IR), the most prominent line that is influenced by the wind and consequently by $\dot{M}$ is $\operatorname{Br} \gamma$, which shows an emission component preferentially in the O-star models.

For the galactic grid, we assume solar abundances as derived by Asplund et al. (2009). In the LMC and SMC models, we adopt the abundances obtained by Hunter et al. (2007) and Trundle et al. (2007) for C, N, O, Mg, Si, and Fe. For P and S, we use the corresponding solar abundances, scaled to the metallicity of the LMC and SMC by a factor of $1 / 2$ and $1 / 7$, respectively. The hydrogen mass fraction is set to $X_{\mathrm{H}}=0.74$ in all models.

In the comoving-frame calculations of the LMC and MW grid models, a micro turbulent velocity of $\xi=10 \mathrm{~km} \mathrm{~s}^{-1}$ is used, while the SMC models are calculated with $\xi=14 \mathrm{~km} \mathrm{~s}^{-1}$.

\subsection{Data products}

The most important output of the model calculations are the synthetic line spectra. We provide a continuous coverage from the UV to the near-IR (NIR) ( $920 \AA-2.4 \mu \mathrm{m})$, including the $K$-band, as well as a significant fraction of the mid-IR domain $(10-20 \mu \mathrm{m})$. These emergent spectra are calculated in the observer's frame and have a spectral resolution of about $R=$ 160.000 (corresponding to $5 \mathrm{~km} \mathrm{~s}^{-1}$ in the velocity space). Fluxcalibrated and continuum-normalized spectra are available. The normalized line spectra of two exemplary wavelength ranges with prominent metal lines are displayed in Fig. 3. This figure illustrates the spectral differences between late O-type giants at different metallicities by comparing models with the same $T_{*}$, $\log g_{\text {grav }}$, and $\dot{M}$ from the different models grids.

We also provide spectral energy distributions (SEDs) over the whole spectrum. These SEDs include all lines but are on a coarse wavelength grid and were calculated in the comoving frame. The SEDs for the three models depicted in Fig. 3 are plotted in Fig. 4 in comparison to a back body with $T_{\text {eff }}=36 \mathrm{kK}$.

Feedback parameters such as the number of hydrogen ionizing and helium ionizing photons and Zanstra temperatures are

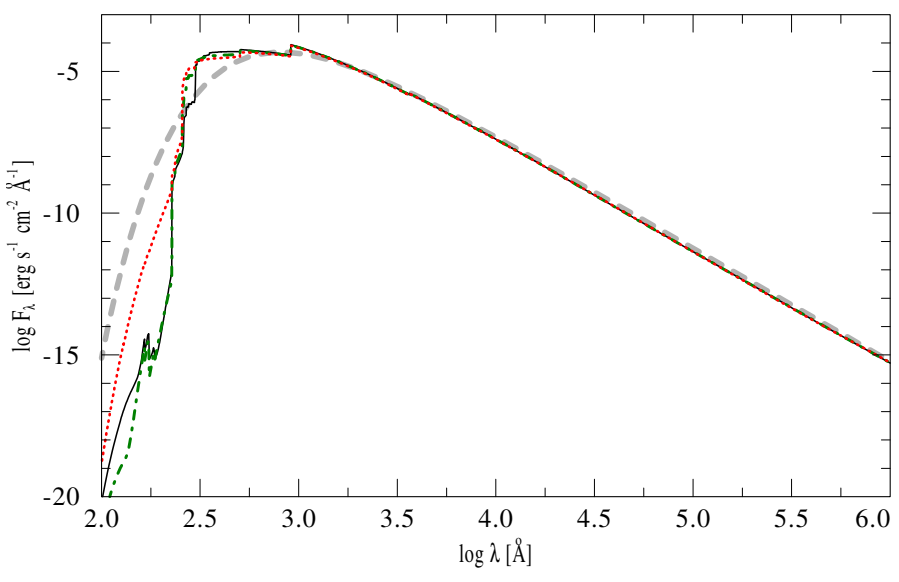

Fig. 4. Spectral energy distributions of the three models shown in Fig. 3 in comparison with a black body of the same effective temperature as the models. The models are plotted with the same line styles and colors as in Fig. 3, while the black body is depicted by a thick gray dashed line.

available for all models. In addition, we provide Johnson $U, B$, and $V$ magnitudes and Stroemgren $u, v, b$, and $y$ magnitudes. In Table 1, the predicted magnitudes and feedback parameters are listed, exemplary for the models shown in Figs. 3 and 4.

The atmospheric structure (e.g., the density and the velocity stratification) is supplied for all models. As an example, the structure information of the model with $T_{*}=25 \mathrm{kK}$ and $\log g_{\text {grav }}=3.2[\mathrm{cgs}]$ from the LMC model grid is listed in Table A.2.

\subsection{The web interface}

All information described in Sect. 3.1 can be accessed via the PoWR web interface ${ }^{2}$. A general description of the interface and how to use it can be found in Todt et al. (2015). Recently, an

2 www.astro.physik.uni-potsdam.de/PowR 
Table 1. Feedback parameters and magnitudes of the three models from Figs. 3-5.

\begin{tabular}{llll}
\hline \hline & MW & LMC & \multicolumn{1}{c}{ SMC } \\
\hline $\log Q_{\mathrm{H}}\left(\mathrm{s}^{-1}\right)$ & 48.83 & 48.82 & 48.82 \\
$T_{\mathrm{Zanstra}, H}(\mathrm{kK})$ & 35.5 & 35.3 & 35.4 \\
$\log Q_{\mathrm{He}}\left(\mathrm{s}^{-1}\right)$ & 47.80 & 47.78 & 47.81 \\
$\log Q_{\mathrm{He} \text { II }}\left(\mathrm{s}^{-1}\right)$ & $-{ }^{b}$ & $-b$ & 42.06 \\
$T_{\mathrm{Zanstra}, H e}(\mathrm{kK})$ & $-{ }^{b}$ & $-{ }^{b}$ & 27.9 \\
$M_{\mathrm{U}}(\mathrm{mag})$ & -6.60 & -6.58 & -6.54 \\
$M_{\mathrm{B}}(\mathrm{mag})$ & -5.42 & -5.41 & -5.37 \\
$M_{\mathrm{V}}(\mathrm{mag})$ & -5.12 & -5.11 & -5.08 \\
$M_{\mathrm{u}}(\mathrm{mag})^{a}$ & -5.52 & -5.50 & -5.46 \\
$M_{\mathrm{b}}(\mathrm{mag})^{a}$ & -5.23 & -5.23 & -5.19 \\
$M_{\mathrm{V}}(\mathrm{mag})^{a}$ & -5.31 & -5.31 & -5.27 \\
$M_{\mathrm{y}}(\mathrm{mag})^{a}$ & -5.14 & -5.14 & -5.10
\end{tabular}

Notes. ${ }^{(a)}$ Stroemgren magnitudes. ${ }^{(b)}$ For these relatively cool models, the Heii Ionizing flux $(\lambda<228 \AA)$ is neglidgeble.

option to obtain the tabulated atmospheric structure and the possibility to download the selected data product for a whole grid was added to the online interface. Both these options are available after having selected a specific model from the grids. More detailed information such as the population numbers or highresolution SEDs calculated in the observer's frame are currently not accessible via the web interface, but can be provided on individual request.

\section{Discussion}

Figure 3 reveals how the metal lines become weaker with decreasing metallicity. A close inspection of this figure, however, also shows that the equivalent widths of the He II lines are decreasing with $Z$. A zoom on the He II $\lambda 4542$ line and the $\mathrm{He}_{\mathrm{I}} \lambda 4713$ line is depicted in Fig. 5, revealing that as the He II lines get weaker the He I lines simultaneously become stronger with decreasing $Z$. Although this effect is relatively small for the He II lines, it can have a noticeable impact on the parameters that one would deduce from spectral line fits using these models. This effect is not limited to the helium lines. Test calculations revealed that it is a general trend that is also displayed by other elements. For example, if the carbon abundance is kept constant but the iron abundance is changed from its default values in the grids to zero, the same effect is also visible in the carbon lines. We are confident that this is not a PoWR specific artefact, since the same effect is also shown by the TLUSTY models calculated by Lanz \& Hubeny (2003).

The reason for the observed dependence of the He I to He II line ratios on metallicity is the changing flux level in the UV and extreme UV, which depends on the metal abundances used in the model calculations. According to the flux level, the ionization structure of the models shifts to a different balance because of the extreme non-LTE situation within the atmospheres of these stars. This leads to the observed differences in the emergent He spectra. Despite this general mechanism, it was not possible to identify specific wavelength ranges or specific transitions that might be chiefly responsible for the observed change in the ionization stratification. Because of the millions of transitions and the various non-LTE effects involved, this is a very difficult task; it is beyond the scope of this paper but deserves a specific study.
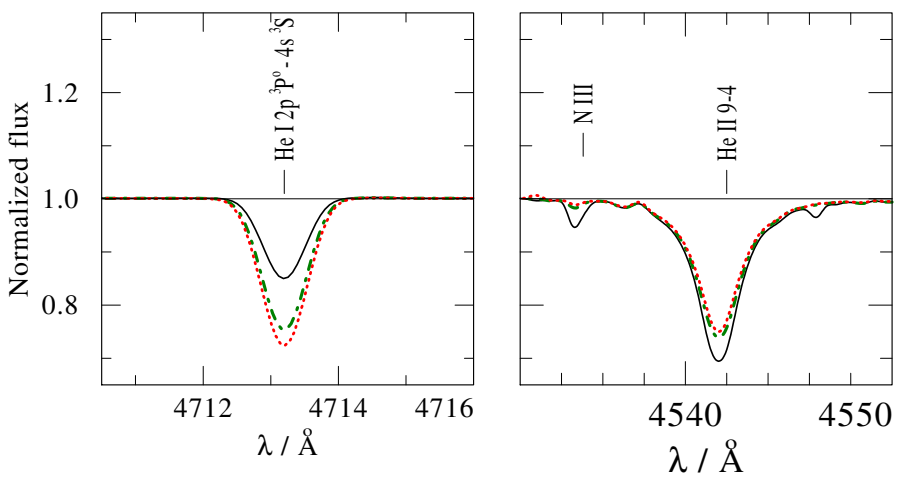

Fig. 5. As in Fig. 3 but showing zooms on the He I $\lambda 4713$ and He II $\lambda 4542$ line.

Massive stars are found to have an earlier spectral type and appear to be younger at low metallicities compared to their solar companions (e.g., Massey et al. 2004; Martins et al. 2004; Mokiem et al. 2004; Crowther \& Hadfield 2006). This is because the stars are considered to be more compact at low $Z$. The finding, illustrated in Fig. 5, might appear to contradict this canonical perception. However, the effect presented here is a different one, since the models have the same $R_{*}$ and the difference between the $T_{2 / 3}$ (effective temperature at $\tau=2 / 3$ ) values of these models is negligible. Using our models to analyze stars would actually also result in higher temperatures at low $Z$ compared to solar metallicities. To understand why, one may imagine two stars, one from the MW and one from the SMC, that have the same spectral type and that exhibit the same equivalent widths in the He I and He II lines. If those lines were to be reproduced by a MW model with a certain stellar temperature, the corresponding model from the SMC grid would not fit to the observations. To reproduce the spectra with a model from the SMC grid, one actually would have to choose a model with a higher $T_{*}$ compared to the MW grid to compensate for the weaker He II and stronger He I lines.

The observed changes in the He spectra with the metallicity highlights the need for non-LTE atmosphere models for the spectral analyses of not only OB-type stars but in principle all hot stars. This is also evident from Fig. 4, which compares the SEDs of the models shown in Fig. 3 with a black body of the same effective temperature. While the flux of the models in the IR and beyond is approximated quite well by the black body, the deviations in the UV and extreme UV are huge. The black-body SED overestimates the number of hydrogen ionizing photons $(\lambda<912 \AA)$ by almost $50 \%$ in the selected examples for all three metallicities. The number of He $\mathrm{I}$ ionizing photons $(\lambda<504 \AA)$ is very low in our detailed models, because these photons are mainly absorbed within the atmosphere and cannot emerge. The black body therefore over-estimates their number by orders of magnitude. The models selected as examples in Figs. 3-5 and Table 1 are not hot enough to emit photons that can ionize He II $(\lambda<228 \AA)$. However, for the hottest models in our grids such photons are predicted in significant number. Of course, black bodies completely fail to approximate this part of the spectrum. All these examples show that the SEDs of massive stars cannot be approximated with black bodies. Instead, sophisticated stellar atmosphere models are required for the investigation of the radiative feedback of massive stars (see e.g., Unsoeld 1968 and Mihalas 1978 for details on stellar atmospheres and the physical background).

The mass of a star, $M_{\text {spec }}$, can be derived spectroscopically by fitting the synthetic spectrum to the wings of pressure-broadened lines. In the case of $\mathrm{OB}$ stars, the Balmer lines are specially 


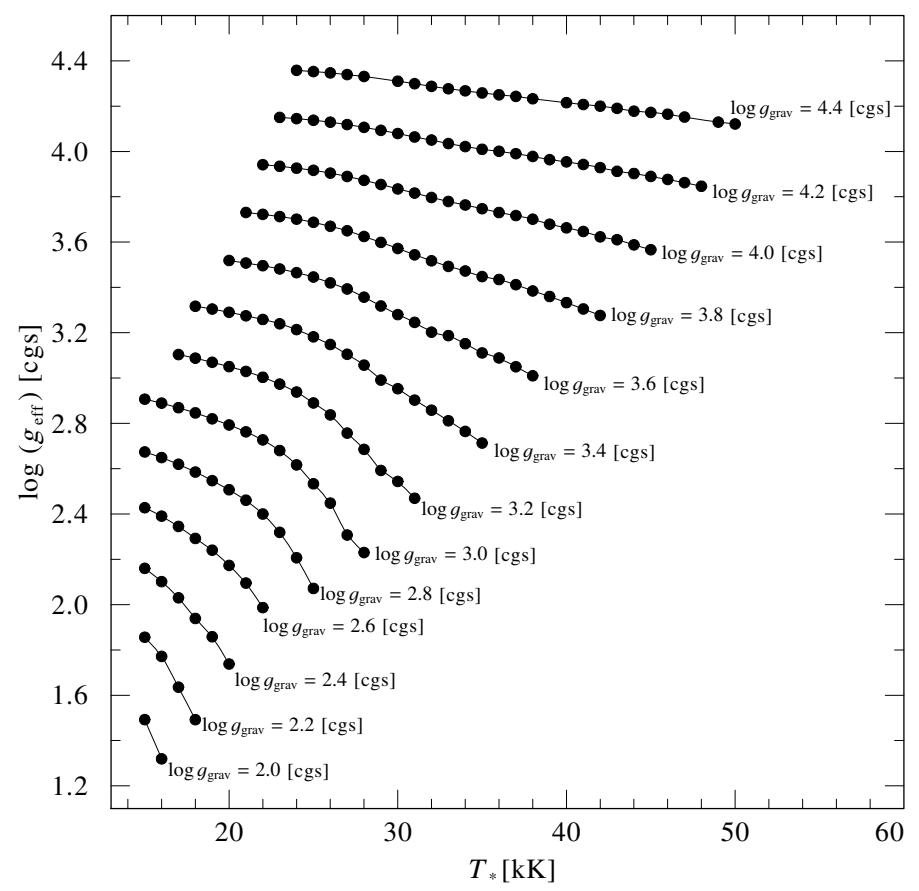

Fig. 6. $\log g_{\text {eff }}-T_{*}$ plane of the LMC model grid illustrating the effect of the radiation pressure on the effective surface gravity. Each black dot refers to one grid model. The thin lines connect models with the same $\log g_{\text {grav }}$.

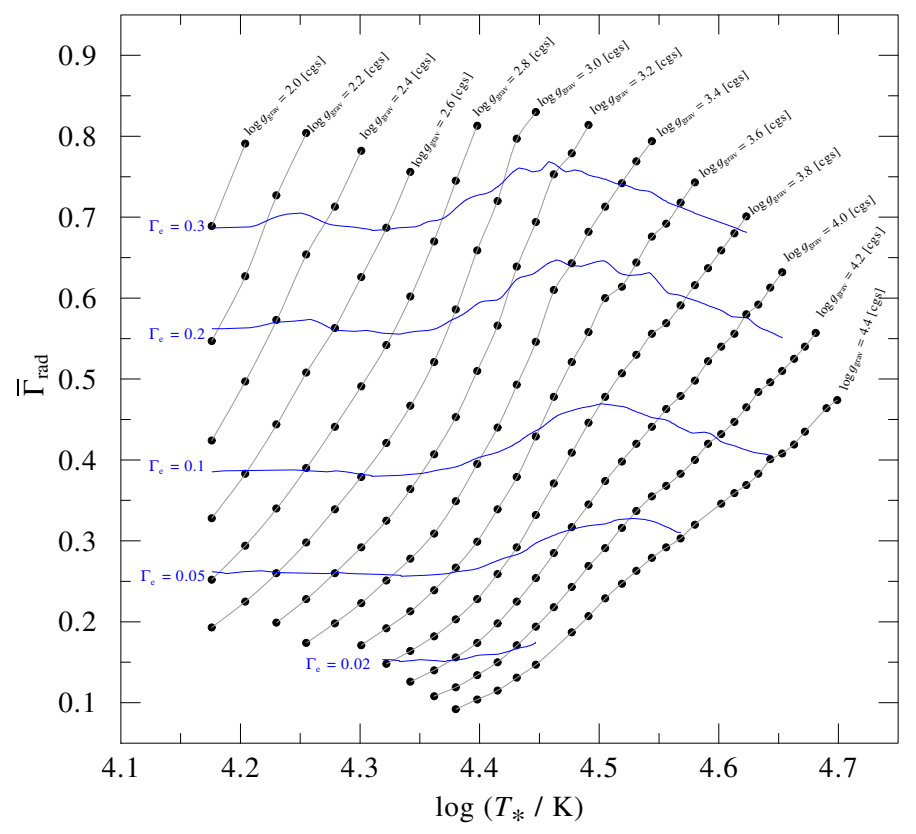

Fig. 7. Eddington Gamma $\bar{\Gamma}_{\text {rad }}$ (black dots) plotted vs. stellar temperature on a logarithmic scale for the models from the LMC grid. The thin black lines connect models with the same $\log g_{\text {grav }}$, while the blue contours depict lines of constant classical Eddington Gamma $\Gamma_{\mathrm{e}}$, as labeled.

suitable for this purpose. The shape and strength of these line wings depend on the electron pressure at their formation depth, which in $\mathrm{OB}$ star atmospheres is located in the lower, quasihydrostatic part of the atmosphere. However, it is not only gravity that enters the hydrostatic equation. In fact, the atmospheric pressure is determined by the effective gravity $g_{\text {eff }}$, which is the gravitational acceleration reduced by the effect of the outwarddirected radiation pressure.
Hence, the quantity which is measured from fitting the line wings is $g_{\text {eff }}$ (see Eq. (5)), and only with the proper correction for the radiation pressure can the correct spectroscopic mass be obtained. The relation between $g_{\text {eff }}$ and $g_{\text {grav }}$ can be investigated from our model grids. In Fig. 6 we plot the effective surface gravity versus the stellar temperature of the models from the LMC grid. The effective surface gravity accounts for the full radiation pressure and is given by

$g_{\mathrm{eff}}=g_{\text {grav }}\left(1-\bar{\Gamma}_{\mathrm{rad}}\right)$,

where $g_{\text {grav }}$ is given by Eq. (3), and $\bar{\Gamma}_{\text {rad }}$ is a weighted mean of the full Eddigton Gamma $\Gamma_{\text {rad }}$ over the hydrostatic domain of the stellar atmosphere as defined by Eq. (27) in Sander et al. (2015). In Fig. 6, models with the same $\log g_{\text {grav }}$ are connected by a thin black line. This figure depicts the difference between $g_{\text {grav }}$ and $g_{\text {eff }}$ throughout the grid. The higher the $L / M$ ratio, the stronger this effect becomes. This is already evident from the definition of the classical Eddington Gamma

$\Gamma_{\mathrm{e}}=\frac{\sigma_{\mathrm{e}}}{4 \pi c G} q_{\text {ion }} \frac{L}{M_{*}}$,

where $q_{\text {ion }}$ is the ionization parameter and $\sigma_{\mathrm{e}}$ denotes the Thomson opacity. Since $q_{\text {ion }}$ is not vastly varying throughout the grid, the variation in $\Gamma_{\mathrm{e}}$ is mainly due to different $L / M$ ratios.

The classical Eddington Gamma $\Gamma_{\mathrm{e}}$ accounts only for the radiative acceleration due to Thomson scattering by free electrons. The full Eddington Gamma $\Gamma_{\text {rad }}$, accounting for all continuum and line opacities, that is, $\Gamma_{\text {rad }}=\Gamma_{\mathrm{e}}+\Gamma_{\text {lines }}+\Gamma_{\text {true cont }}$, is significantly larger than $\Gamma_{\mathrm{e}}$. This is illustrated in Figs. 7 and 8. Figure 7 illustrates the connection between the stellar temperature, the full mean Eddington Gamma $\bar{\Gamma}_{\text {rad }}$, and the classical Eddington Gamma $\Gamma_{\mathrm{e}}$. As in Fig. 6, the models are taken from the LMC grid. Each filled circle refers to one model, while those models with the same $\log g_{\text {grav }}$ are connected by a thin black line. The blue contours in this plot refer to lines of the same $\Gamma_{\mathrm{e}}$. Comparing these contour lines with the $\bar{\Gamma}_{\text {rad }}$ values demonstrates the idea that a low value for the classical Eddington Gamma $\Gamma_{\mathrm{e}}$ does not necessarily mean that a star is far from the Eddington limit. This comparison also indicates that the relation between $\bar{\Gamma}_{\text {rad }}$ and $\Gamma_{\mathrm{e}}$ is not linear but quite complex throughout the grid, which is because of the $\bar{\Gamma}_{\text {rad }}$ temperature dependence. This result suggests that stellar properties (e.g., $\dot{M}$ ) should be correlated with $\Gamma_{\text {rad }}$ rather than with $\Gamma_{\mathrm{e}}$.

We therefore derive the dependence of $\bar{\Gamma}_{\text {rad }}$ on $\Gamma_{\mathrm{e}}$. For this purpose, we plot in Fig. 8 the values of $\bar{\Gamma}_{\text {rad }}$ over $\Gamma_{\mathrm{e}}$ for the models from the LMC model grid. The relation between $\Gamma_{\mathrm{e}}$ and $\bar{\Gamma}_{\text {rad }}$ can be best approximated with a fourth-order polynomial of the form

$\bar{\Gamma}_{\mathrm{rad}}=C_{1}+C_{2} \Gamma_{\mathrm{e}}+C_{3} \Gamma_{\mathrm{e}}^{2}+C_{4} \Gamma_{\mathrm{e}}^{3}+C_{5} \Gamma_{\mathrm{e}}^{4}$.

The coefficients for the fit are given in Table 2, where we also include the relations derived by means of the models from the SMC and MW grid. The corresponding figures showing the MW and the SMC fit are shown in Appendix B. In comparison to the LMC relation, the fits to the SMC and MW models lie slightly below and above, respectively, revealing that $Z$ has only a modest effect on $\bar{\Gamma}_{\text {rad }}$ within the parameter range studied in this work. While this might sound surprising initially, one must keep in mind that $\bar{\Gamma}_{\text {rad }}$ is only the mean over the hydrostatic domain (see Sander et al. 2015) and does not cover the wind where the influence of $Z$ might be much larger. 


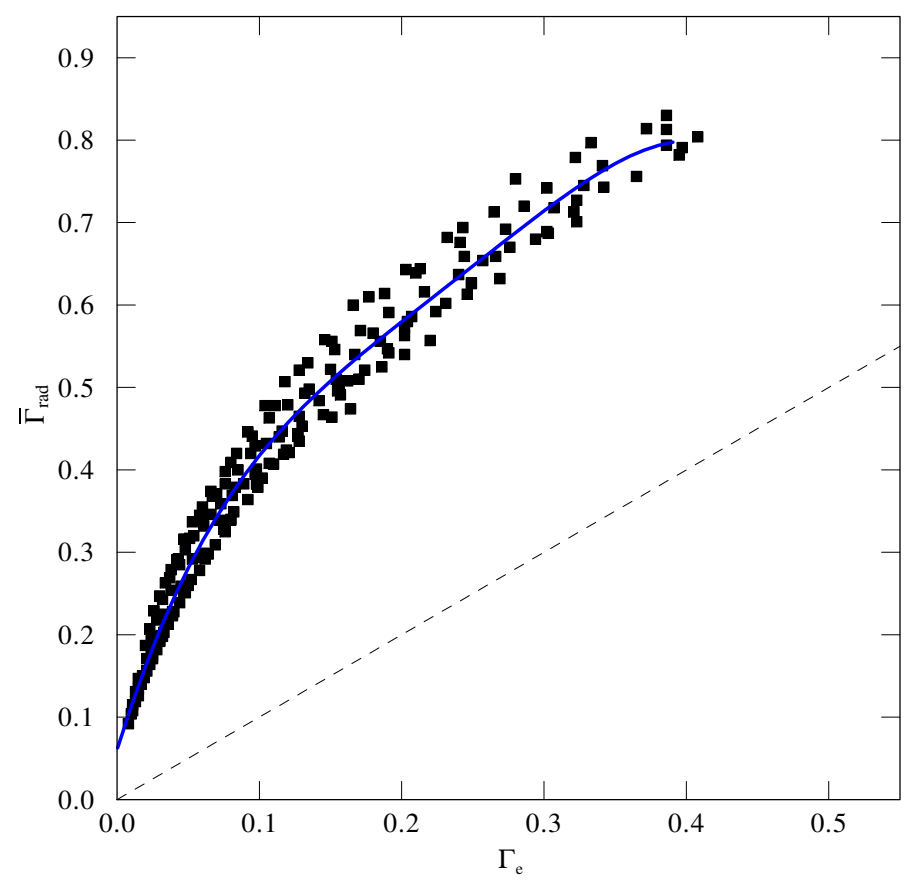

Fig. 8. Eddington Gamma $\bar{\Gamma}_{\text {rad }}$ as a function of the classical Eddington Gamma $\Gamma_{\mathrm{e}}$. Each symbol refers to one model from the LMC grid. The influence of the model parameter $T_{*}$ and $g_{\text {eff }}$ is limited, as reflected by the modest scatter of the data points. The blue straight line represents the fit to the data points (see Eq. (7) and Table 2). The black dashed line indicates $\bar{\Gamma}_{\text {rad }}=\Gamma_{\mathrm{e}}$, i.e., the radiation pressure would be purely due to electron scattering.

Table 2. Coefficients of relations between $\Gamma_{\mathrm{e}}$ and $\bar{\Gamma}_{\text {rad }}$ (Eq. (7)) for the SMC, LMC, and MW models.

\begin{tabular}{llllll}
\hline \hline Grid & $C_{1}$ & $C_{2}$ & $C_{3}$ & $C_{4}$ & $C_{5}$ \\
\hline SMC & 0.06 & 4.69 & -19.93 & 51.97 & -51.13 \\
LMC & 0.06 & 5.57 & -26.68 & 74.77 & -78.33 \\
MW & 0.08 & 5.26 & -19.88 & 40.06 & -29.65 \\
\hline
\end{tabular}

The effect of the radiation pressure on the Balmer line wings is illustrated in Fig. 9, which shows the spectral region around the $\mathrm{H} \delta$ line for six different models from the LMC grid. These models have the same value for the pure gravitational acceleration of $\log g_{\text {grav }}=2.4$, but exhibit substantial differences in the pressure broadened $\mathrm{H} \delta$ line wings. This is because of the different $\log g_{\text {eff }}$ values that vary between 1.7 [cgs] and 2.2 [cgs] due to the change in the radiation pressure. The variations in the other lines visible in Fig. 9 are mainly attributable to the different stellar temperatures of the models.

As shown above for $\bar{\Gamma}_{\text {rad }}$, the impact of the metallicity on the density structure and the pressure broadening of the spectral lines is quite weak in the metallicity domain explored in this work. This is illustrated by Fig. 10 that displays the same spectral range as Fig. 9, while it depicts the models shown in Fig. 3. These models exhibit the same stellar parameters, but were calculated for MW, LMC, and SMC metallicity. The small difference between the wings of the $\mathrm{H} \delta$ line exemplifies the limited effect of the metallicity.

\section{Potential applications}

We have presented extensive atmosphere model grids for OBtype stars calculated with the PoWR code for MW, LMC, and

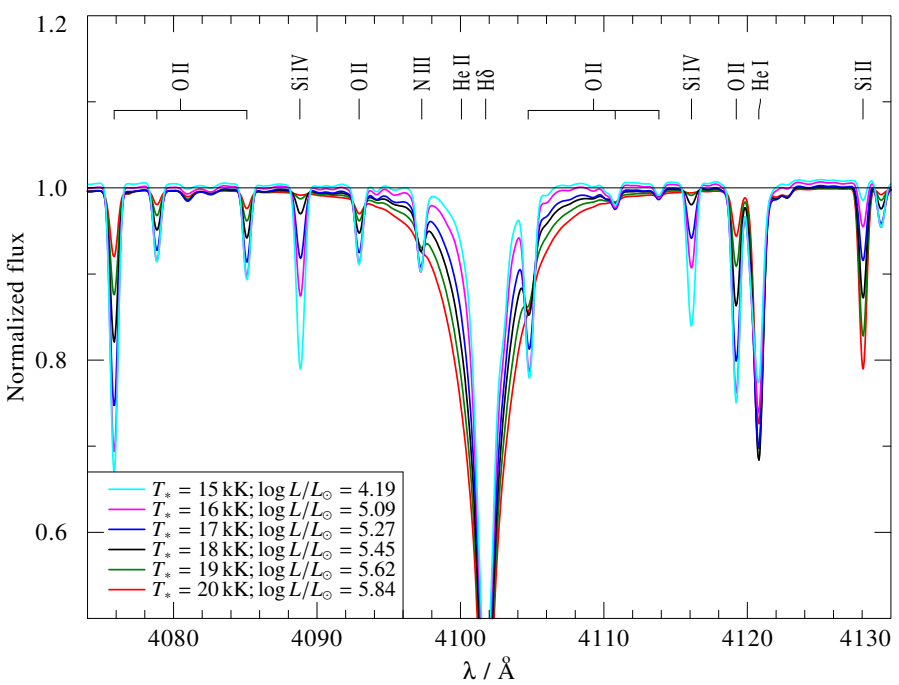

Fig. 9. Normalized spectra of six models from the LMC grid showing the spectral range around the $\mathrm{H} \delta$ line. All models have the same $\log g_{\text {grav }}$ but different $T_{*}$ and $L$. See inlet for details.

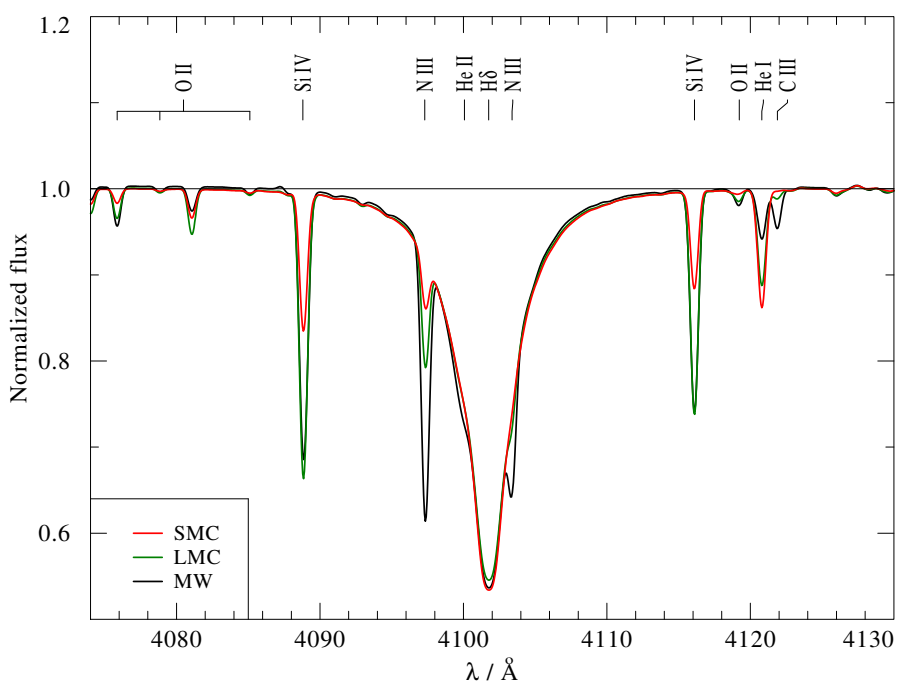

Fig. 10. Like Fig. 9 but for $\mathrm{H} \delta$, showing the models underlying Figs. 3 and 5.

SMC metallicities. Altogether 785 models have been calculated for four model grids. Two grids are available for SMC metallicities, while one grid has so far been calculated for the MW and another for the LMC. Further grids extending the parameter space, especially with respect to the mass-loss rate, are in preparation and will be the subject of a forthcoming paper discussing calibrations between spectral types and physical parameters.

Based on these models, we have illustrated the impact of the radiation pressure on the surface gravity and on the emergent spectra. We derived approximate relations between the classical Eddington Gamma, accounting only for scattering by free electrons, and the full Eddington Gamma, which takes all continuum and line opacities into account.

The immediate application of the model grids provided here is for quantitative spectral analyses. Such analysis proceeds in two steps. First, the observed (normalized or flux-calibrated) line spectrum is fitted to the synthetic spectra from the grid. The stellar temperature can be deduced by fitting the helium and metal lines, paying special attention to the temperature-sensitive ratios between lines of different ionization stages. The surface gravity 
is adjusted by fitting the pressure-broadened profiles, especially of the hydrogen and helium lines. The turbulent and rotational contribution to the line broadening must be separated, for example with the iacob-broad tool (Simón-Díaz \& Herrero 2014) applied to narrow metal lines.

The UV resonance lines, and possibly the strongest lines in the optical (e.g., $\mathrm{H} \alpha$ ) might form in the stellar wind; comparison with the grids calculated for different mass-loss rates may thus give a constraint to this parameter.

As the second step, the luminosity of the star is determined from fitting the model SED to flux-calibrated spectra and/or filter photometry. Here, the model flux has to be scaled according to the distance of the star, that is, knowledge of the distance is essential here. At the same time, the interstellar reddening and extinction need to be accounted for, for example by modifying the model SED by means of a reddening law, so that the shape of the observed SED is reproduced. Thus, this procedure allows to simultaneously derive the luminosity of a star and the interstellar reddening along the line of sight.

Besides spectra and SEDs, further model predictions such as feedback parameters and atmospheric stratifications are provided online for all models as well. These model grids allow a wide range of applications, from spectral analyses to theoretical studies that require atmospheric stratifications of atomic population numbers as input.

Acknowledgements. We thank the anonymous referee for their constructive comments. A. A.C.S. is supported by the Deutsche Forschungsgemeinschaf (DFG) under grant HA 1455/26. V. R. is grateful for financial support from the Deutsche Akademische Austauschdienst (DAAD) as part of the Graduate School Scholarship Program. T.S. and L. M. O. acknowledge support from the german "Verbundforschung" (DLR) grants, 50 OR 1612 and 50 OR 1508, respectively.

\section{References}

Almeida, L. A., Sana, H., Taylor, W., et al. 2017, A\&A, 598, A84 Asplund, M., Grevesse, N., Sauval, A. J., \& Scott, P. 2009, ARA\&A, 47, 481 Bromm, V., \& Larson, R. B. 2004, ARA\&A, 42, 79

Brott, I., de Mink, S. E., Cantiello, M., et al. 2011, A\&A, 530, A115

Castor, J. I., \& Lamers, H. J. G. L. M. 1979, ApJS, 39, 481

Crowther, P. A., \& Hadfield, L. J. 2006, A\&A, 449, 711

Crowther, P. A., \& Walborn, N. R. 2011, MNRAS, 416, 1311

de Mink, S. E., \& Mandel, I. 2016, MNRAS, 460, 3545

Dufour, R. J., Shields, G. A., \& Talbot, Jr., R. J. 1982, ApJ, 252, 461

Ekström, S., Georgy, C., Eggenberger, P., et al. 2012, A\&A, 537, A146

Eldridge, J. J., \& Stanway, E. R. 2016, MNRAS, 462, 3302

Eldridge, J. J., Stanway, E. R., Xiao, L., et al. 2017, PASA, 34, e058

Foellmi, C., Moffat, A. F. J., \& Guerrero, M. A. 2003, MNRAS, 338, 360

Gräfener, G., Koesterke, L., \& Hamann, W.-R. 2002, A\&A, 387, 244

Hainich, R., Rühling, U., Todt, H., et al. 2014, A\&A, 565, A27

Hainich, R., Pasemann, D., Todt, H., et al. 2015, A\&A, 581, A21

Hainich, R., Oskinova, L. M., Shenar, T., et al. 2018, A\&A, 609, A94

Hamann, W.-R. 1981, A\&A, 93, 353

Hamann, W.-R., \& Gräfener, G. 2003, A\&A, 410, 993

Hamann, W.-R., \& Gräfener, G. 2004, A\&A, 427, 697
Hamann, W.-R., \& Koesterke, L. 1998, A\&A, 335, 1003

Hamann, W.-R., Koesterke, L., \& Wessolowski, U. 1995, A\&A, 299, 151

Hillier, D. J. 1991, A\&A, 247, 455

Hillier, D. J., \& Miller, D. L. 1998, ApJ, 496, 407

Hubeny, I., \& Lanz, T. 1995, ApJ, 439, 875

Humphreys, R. M., \& Davidson, K. 1994, PASP, 106, 1025

Hunter, I., Dufton, P. L., Smartt, S. J., et al. 2007, A\&A, 466, 277

Kudritzki, R. P., Pauldrach, A., Puls, J., \& Abbott, D. C. 1989, A\&A, 219, 205

Kudritzki, R.-P., Hummer, D. G., Pauldrach, A. W. A., et al. 1992, A\&A, 257, 655

Lamers, H. J. G. L. M., \& Cassinelli, J. P. 1999, Introduction to Stellar Winds, 452

Lamers, H. J. G. L. M., Snow, T. P., \& Lindholm, D. M. 1995, ApJ, 455, 269

Lanz, T., \& Hubeny, I. 2003, ApJS, 146, 417

Lanz, T., \& Hubeny, I. 2007, ApJS, 169, 83

Larsen, S. S., Clausen, J. V., \& Storm, J. 2000, A\&A, 364, 455

Leitherer, C., Robert, C., \& Drissen, L. 1992, ApJ, 401, 596

Leitherer, C., Ekström, S., Meynet, G., et al. 2014, ApJS, 212, 14

Marchant, P., Langer, N., Podsiadlowski, P., Tauris, T. M., \& Moriya, T. J. 2016, A\&A, 588, A50

Martins, F., Schaerer, D., Hillier, D. J., \& Heydari-Malayeri, M. 2004, A\&A, 420, 1087

Massey, P., Bresolin, F., Kudritzki, R. P., Puls, J., \& Pauldrach, A. W. A. 2004, ApJ, 608, 1001

Meynet, G., \& Maeder, A. 2005, A\&A, 429, 581

Mihalas, D. 1978, Stellar atmospheres /2nd edition (San Francisco: W. H. Freeman and Co.), 650

Mokiem, M. R., Martín-Hernández, N. L., Lenorzer, A., de Koter, A., \& Tielens, A. G. G. M. 2004, A\&A, 419, 319

Mokiem, M. R., de Koter, A., Vink, J. S., et al. 2007, A\&A, 473, 603

Pauldrach, A., Puls, J., \& Kudritzki, R. P. 1986, A\&A, 164, 86

Puls, J. 2008, Massive Stars as Cosmic Engines, eds. F. Bresolin, P. A. Crowther, \& J. Puls, IAU Symp., 250, 25

Puls, J., Kudritzki, R.-P., Herrero, A., et al. 1996, A\&A, 305, 171

Puls, J., Vink, J. S., \& Najarro, F. 2008, A\&ARv, 16, 209

Ramachandran, V., Hainich, R., Hamann, W.-R., et al. 2018a, A\&A, 609, A7

Ramachandran, V., Hamann, W.-R., Hainich, R., et al. 2018b, A\&A, 615, A40

Runacres, M. C., \& Owocki, S. P. 2002, A\&A, 381, 1015

Sana, H., de Koter, A., de Mink, S. E., et al. 2013, A\&A, 550, A107

Sander, A., Hamann, W.-R., \& Todt, H. 2012, A\&A, 540, A144

Sander, A., Shenar, T., Hainich, R., et al. 2015, A\&A, 577, A13

Sander, A. A. C., Hamann, W.-R., Todt, H., Hainich, R., \& Shenar, T. 2017, A\&A, 603, A86

Schneider, F. R. N., Sana, H., Evans, C. J., et al. 2018, Science, 359, 69

Shenar, T., Oskinova, L., Hamann, W.-R., et al. 2015, ApJ, 809, 135

Shenar, T., Hainich, R., Todt, H., et al. 2016, A\&A, 591, A22

Shenar, T., Richardson, N. D., Sablowski, D. P., et al. 2017, A\&A, 598, A85

Simón-Díaz, S., \& Herrero, A. 2014, A\&A, 562, A135

Smith, L. F. 1968, MNRAS, 140, 409

Smith, L. F., Shara, M. M., \& Moffat, A. F. J. 1996, MNRAS, 281, 163

Šurlan, B., Hamann, W.-R., Kubát, J., Oskinova, L., \& Feldmeier, A. 2012a, Proceedingsof a Scientific Meeting in Honor of Anthony F. J. Moffat, eds. L. Drissen, C. Robert, N. St-Louis, \& A. F. J. Moffat, ASP Conf. Ser., 465, 134

Šurlan, B., Hamann, W.-R., Kubát, J., Oskinova, L. M., \& Feldmeier, A. 2012b, A\&A, 541, A37

Todt, H., Sander, A., Hainich, R., et al. 2015, A\&A, 579, A75

Trundle, C., Dufton, P. L., Hunter, I., et al. 2007, A\&A, 471, 625

Unsoeld, A. 1968, Physik der Sternatmosphaeren MIT besonderer Beruecksichtigung (der Sonne)

van Genderen, A. M. 2001, A\&A, 366, 508 
R. Hainich et al.: PoWR grids of non-LTE model atmospheres for OB-type stars of various metallicities

\section{Appendix A: Additional tables}

Table A.1. Atomic model used to construct the OB-type model grids.

\begin{tabular}{|c|c|c|c|c|c|}
\hline Ion & Number of levels & Number of lines ${ }^{a}$ & Ion & Number of levels & Number of lines ${ }^{a}$ \\
\hline $\mathrm{H}_{\mathrm{I}}$ & 22 & 231 & $\mathrm{~S} v$ & 10 & 8 \\
\hline $\mathrm{H}_{\text {II }}$ & 1 & 0 & S VI & 1 & 0 \\
\hline $\mathrm{He}_{\mathrm{I}}$ & 35 & 271 & $\mathrm{Mg}_{\mathrm{I}}$ & 1 & 0 \\
\hline He II & 26 & 325 & $\operatorname{Mg}_{\text {II }}$ & 32 & 120 \\
\hline He III & 1 & 0 & $\mathrm{Mg}$ III & 43 & 158 \\
\hline $\mathrm{N}_{\mathrm{I}}$ & 10 & 13 & Mg IV & 17 & 27 \\
\hline $\mathrm{N}_{\text {II }}$ & 38 & 201 & $\mathrm{Mg} \mathrm{v}$ & 20 & 25 \\
\hline $\mathrm{N}_{\text {IIII }}^{b}$ & 5685 & 219464 & $\mathrm{Si} \mathrm{I}^{b}$ & 20 & 4345 \\
\hline N IV & 38 & 154 & Si II & 20 & 35 \\
\hline $\mathrm{Nv}$ & 20 & 114 & Si III & 24 & 68 \\
\hline $\mathrm{N}$ VI & 14 & 48 & Si IV & 23 & 72 \\
\hline $\mathrm{C}_{\mathrm{I}}$ & 15 & 30 & Si v & 1 & 0 \\
\hline $\mathrm{C}_{\text {II }}$ & 32 & 148 & $\mathrm{P}_{\text {IV }}$ & 12 & 16 \\
\hline $\mathrm{C}_{\text {III }}$ & 40 & 226 & $\mathrm{PV}_{\mathrm{V}}$ & 11 & 22 \\
\hline $\mathrm{C}_{\mathrm{IV}}$ & 25 & 230 & $\mathrm{P}_{\text {VI }}$ & 1 & 0 \\
\hline $\mathrm{Cv}$ & 29 & 120 & $\mathrm{G} \mathrm{I}^{c}$ & 1 & 0 \\
\hline $\mathrm{C}_{\mathrm{VI}}$ & 1 & 0 & $\mathrm{G} \mathrm{III}^{c}$ & 3 & 2 \\
\hline $\mathrm{O}_{\mathrm{I}}$ & 13 & 15 & $\mathrm{G}_{\mathrm{III}}{ }^{c}$ & 13 & 40 \\
\hline $\mathrm{O}_{\text {II }}$ & 37 & 150 & $\mathrm{GIV}^{c}$ & 18 & 77 \\
\hline $\mathrm{O}_{\mathrm{III}}$ & 33 & 121 & $\mathrm{G} \mathrm{v}^{c}$ & 22 & 107 \\
\hline O IV & 29 & 76 & $\mathrm{G} \mathrm{VI}^{c}$ & 29 & 194 \\
\hline $\mathrm{Ov}$ & 36 & 153 & $\mathrm{G} \mathrm{VI}^{c}$ & 29 & 194 \\
\hline $\mathrm{O}_{\mathrm{VI}}$ & 16 & 101 & $\mathrm{G} \mathrm{vII}^{c}$ & 19 & 87 \\
\hline O VII & 15 & 64 & $\mathrm{G} \mathrm{vIII}^{c}$ & 14 & 49 \\
\hline$S_{\text {III }}$ & 23 & 38 & $\mathrm{G} \mathrm{IX}^{c}$ & 15 & 56 \\
\hline$S_{\text {IV }}$ & 11 & 13 & & & \\
\hline
\end{tabular}

Notes. ${ }^{(a)}$ Number of lines accounted for during the comoving-frame calculations. ${ }^{(b)}$ For those quantities where two values are given, the second one refers to the Galactic OB-star grid, while the first one is valid for all other grids. ${ }^{(c)} \mathrm{G}$ denotes a generic atom which incorporates the following iron group elements: $\mathrm{Sc}, \mathrm{Ti}, \mathrm{V}, \mathrm{Cr}, \mathrm{Mn}, \mathrm{Fe}, \mathrm{Co}$, and $\mathrm{Ni}$. The corresponding ions are treated by means of a superlevel approach (for details see Gräfener et al. 2002). 


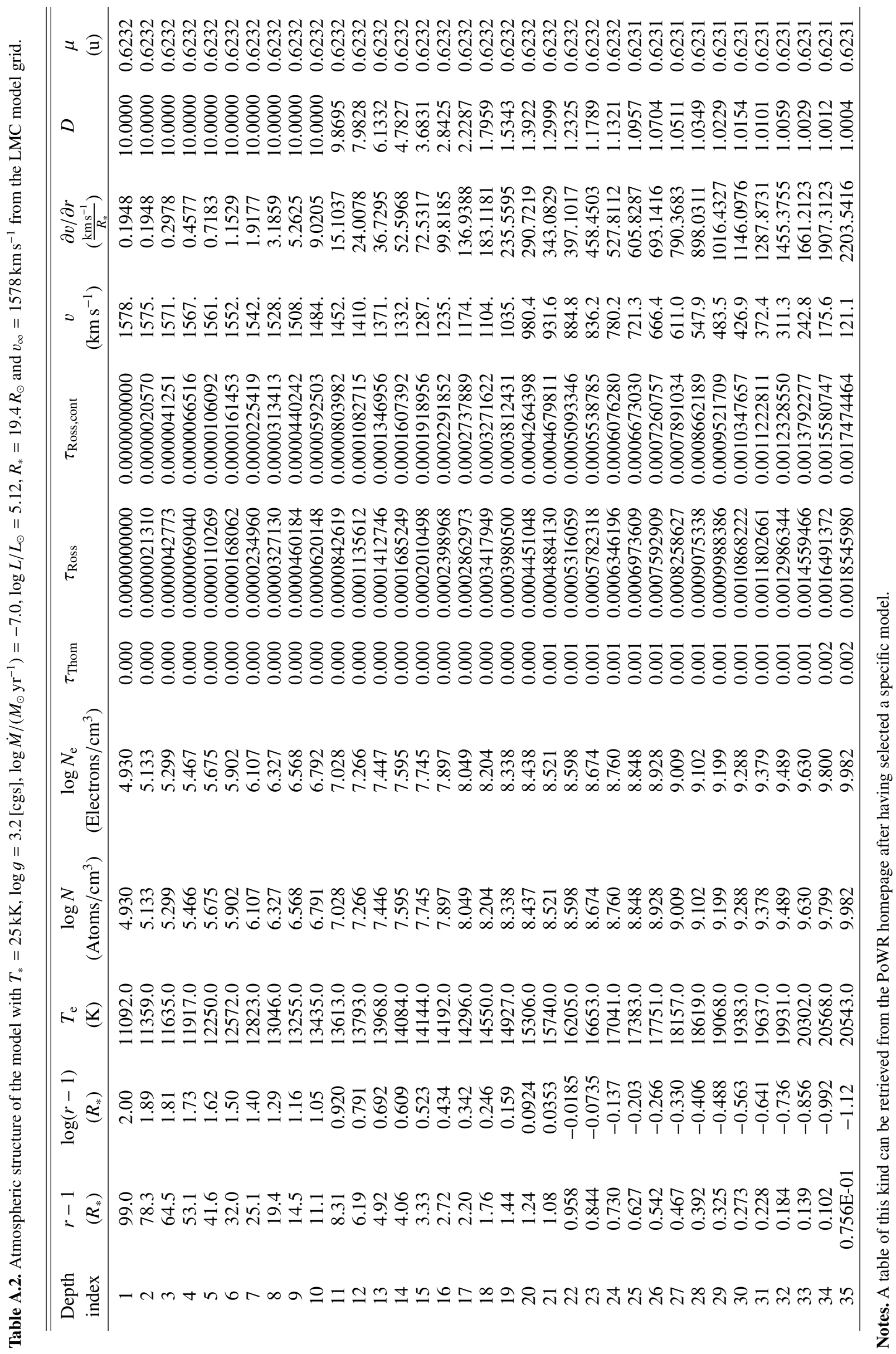


R. Hainich et al.: PoWR grids of non-LTE model atmospheres for OB-type stars of various metallicities

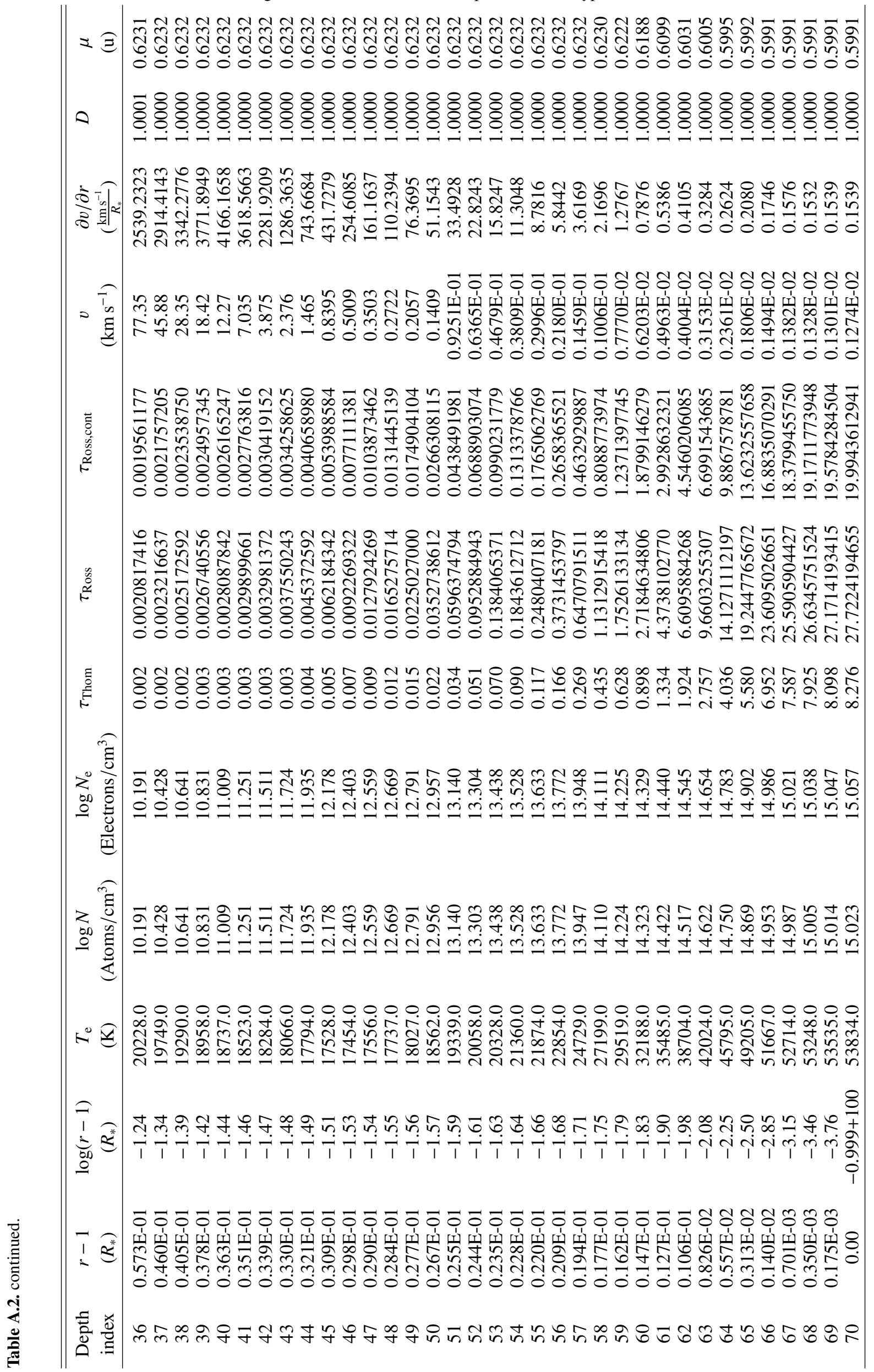




\section{Appendix B: Additional figures}

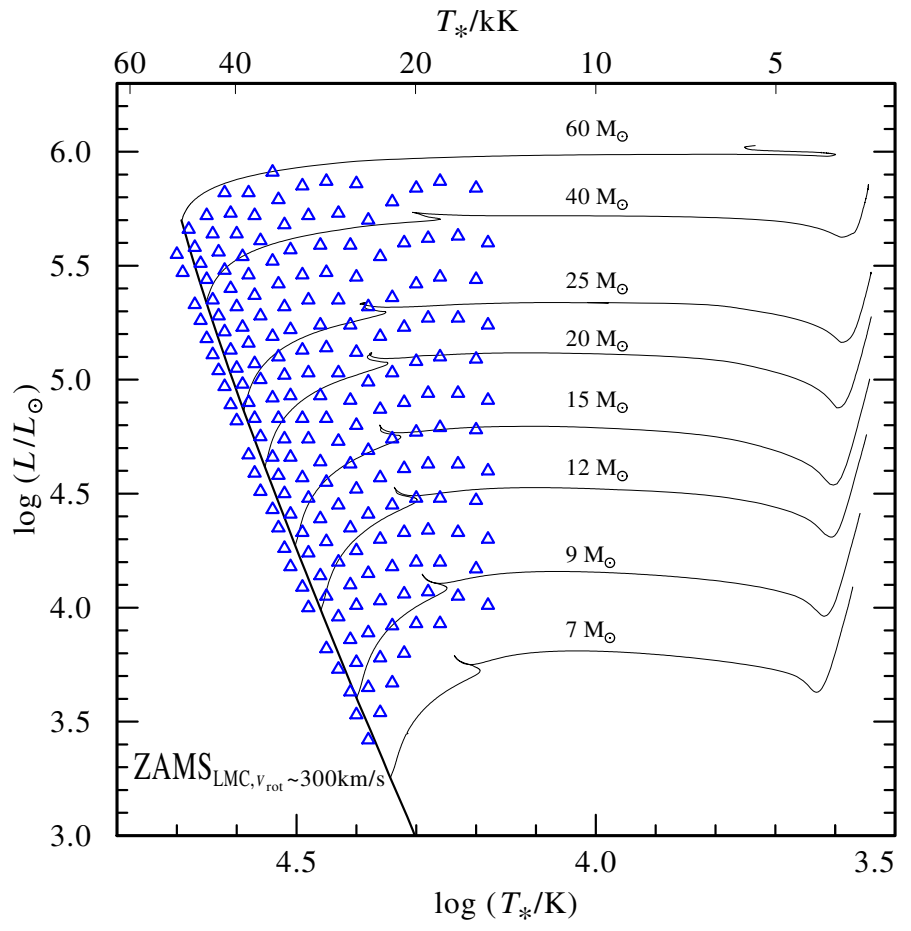

Fig. B.1. Same as Fig. 2 but for the LMC grid.

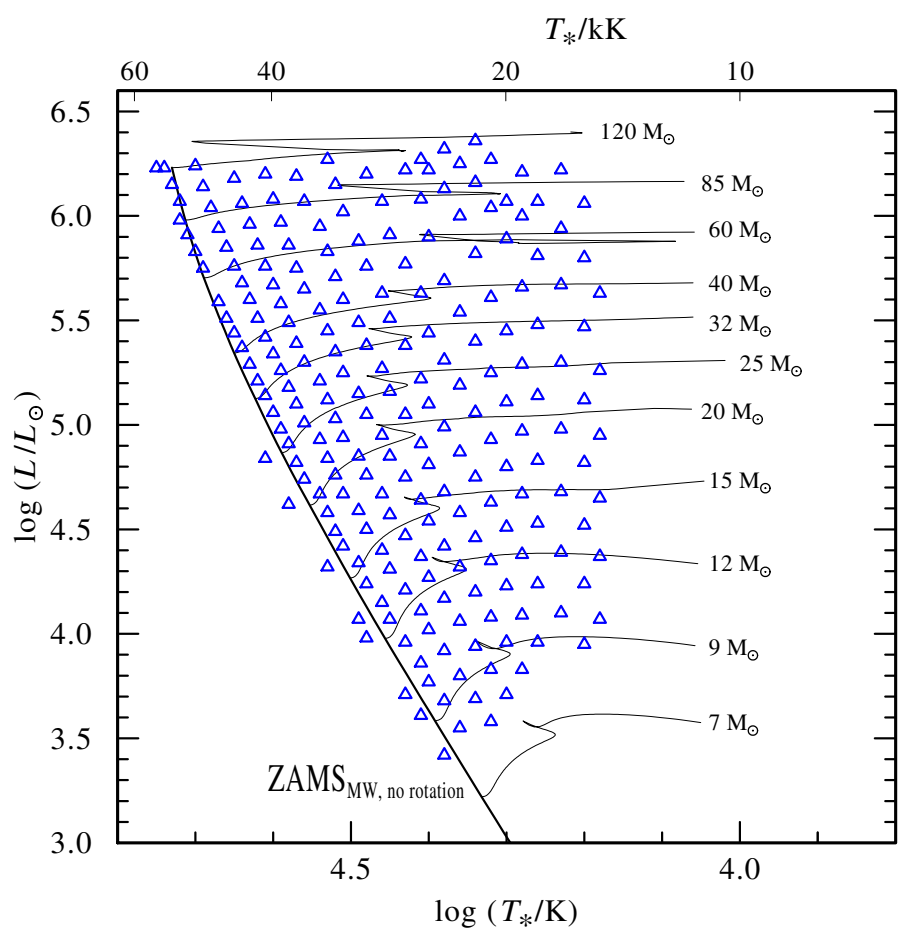

Fig. B.2. Same as Fig. 2 but for the MW grid. The depicted stellar evolution tracks were calculated by Ekström et al. (2012). Only the relevant parts of the tracks are plotted.

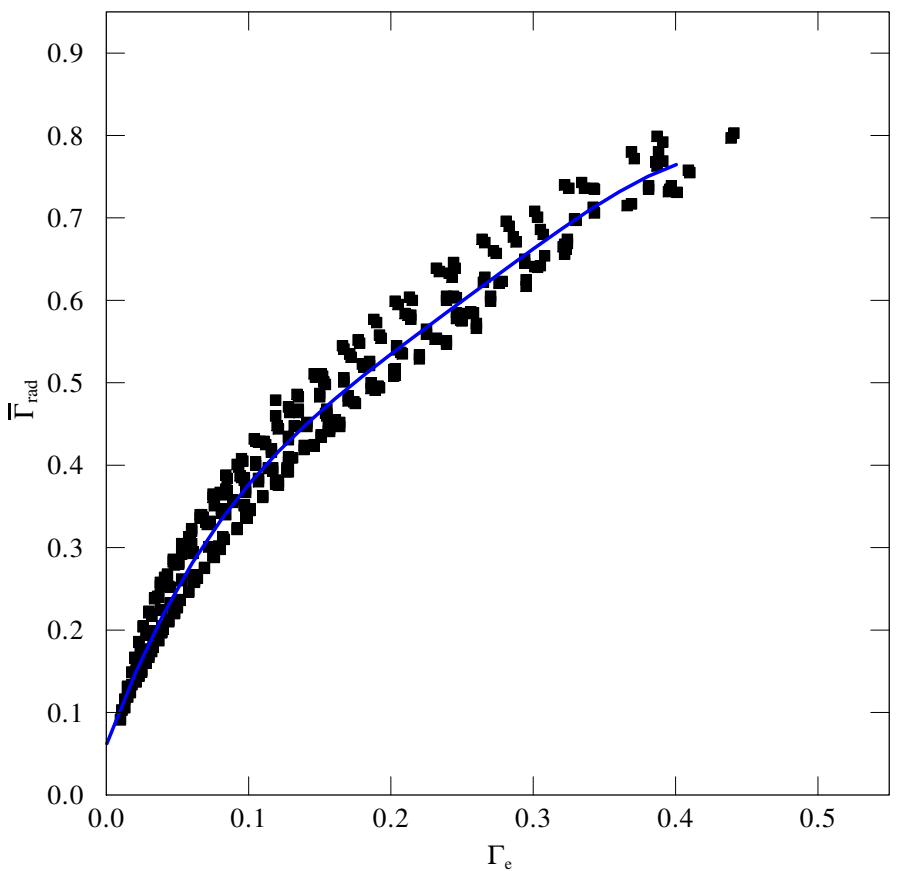

Fig. B.3. Same as Fig. 8 but for the models from the SMC grid.

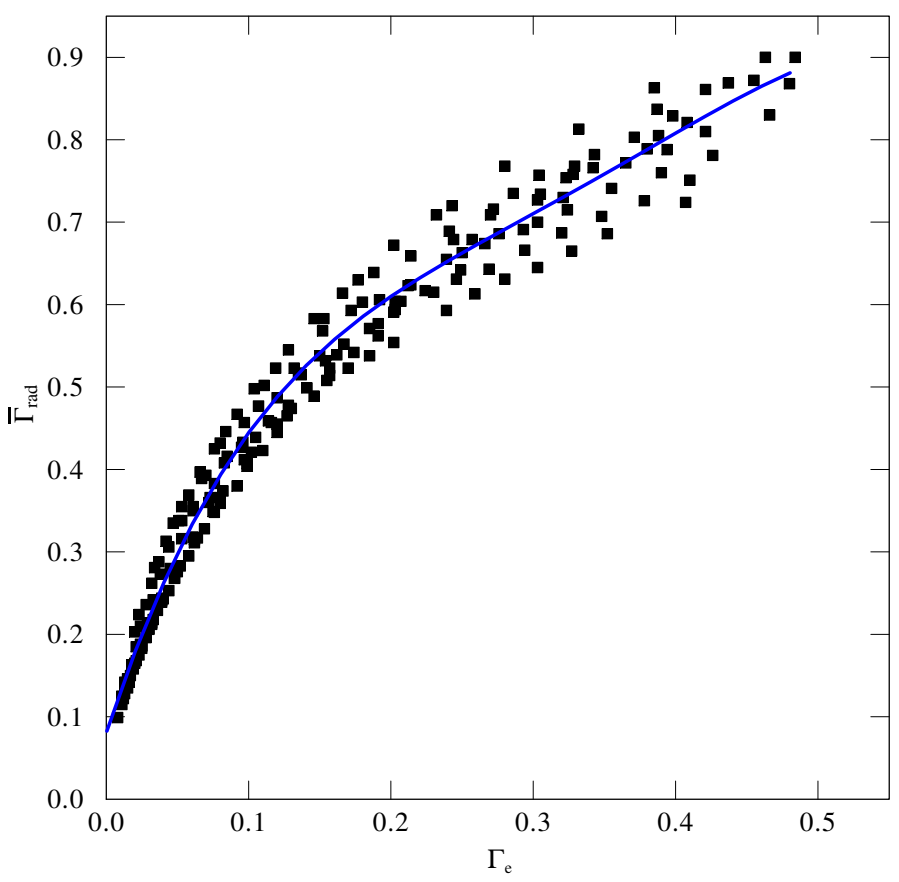

Fig. B.4. Same as Fig. 8 but for the models from the MW grid. 